\title{
Notoginsenoside $\mathbf{R} 1$ ameliorates podocyte injury in rats with diabetic nephropathy by activating the PI3K/Akt signaling pathway
}

\author{
GUODONG HUANG ${ }^{1,2 *}$, JIANZHEN LV ${ }^{3 *}$, TONGYU LI $^{3}$, GUOLI HUAI $^{4}$, XIANG LI $^{4}$, SHAOWEI XIANG $^{1}$, \\ LONGLONG WANG $^{3}$, ZHENLIN QIN $^{3}$, JIANLI PANG ${ }^{1}$, BINGYU ZOU $^{5}$ and YI WANG \\ ${ }^{1}$ Ruikang Hospital Affiliated to Guangxi University of Chinese Medicine, Nanning, Guangxi 530011; \\ ${ }^{2}$ Hepatobiliary and Intestinal Surgery Research Center, Central South University, Changsha, Hunan 410008; \\ ${ }^{3}$ Guangxi University of Chinese Medicine, Nanning, Guangxi 530001; ${ }^{4}$ Department of Biomedical Engineering, \\ Medical School of the University of Electronic Science and Technology of China, Chengdu, Sichuan 610054; \\ Departments of ${ }^{5}$ Gynecology and ${ }^{6}$ Pharmacy, Sichuan Academy of Medical Sciences and \\ Sichuan Provincial People's Hospital, Chengdu, Sichuan 610072, P.R. China
}

Received June 30, 2015; Accepted August 11, 2016

DOI: $10.3892 /$ ijmm.2016.2713

\begin{abstract}
The present study was designed to examine the protective effect of notoginsenoside R1 (NR1) on podocytes in a rat model of streptozotocin (STZ)-induced diabetic nephropathy (DN), and to explore the mechanism responsible for NR1-induced renal protection. Diabetes was induced by a single injection of STZ, and NR1 was administered daily at a dose of $5 \mathrm{mg} / \mathrm{kg}$ (low dose), $10 \mathrm{mg} / \mathrm{kg}$ (medium) and $20 \mathrm{mg} / \mathrm{kg}$ (high) for 16 weeks in Sprague-Dawley rats. Blood glucose levels, body weight and proteinuria were measured every 4 weeks, starting on the day that the rats received NR1. Furthermore, on the day of sacrifice, blood, urine and kidneys were collected in order to assess renal function according to general parameters. Pathological staining was performed to evaluate the renal protective effect of NR1, and the expression of the key slit diaphragm proteins, namely neprhin, podocin and desmin, were evaluated. In addition, the serum levels of inflammatory cytokines [tumor necrosis factor- $\alpha$ (TNF- $\alpha$ ), tumor growth factor- $\beta 1$ (TGF- $\beta 1$ ), interleukin (IL)-1 and IL-6]
\end{abstract}

Correspondence to: Dr Yi Wang, Department of Pharmacy, Sichuan Academy of Medical Sciences and Sichuan Provincial People's Hospital, 32 West Ring Road, Chengdu, Sichuan 610072, P.R. China

E-mail:w_yi@yahoo.com

Dr Bingyu Zou, Department of Gynecology, Sichuan Academy of Medical Sciences and Sichuan Provincial People's Hospital, 32 West Ring Road, Chengdu, Sichuan 610072, P.R. China

E-mail: bingyuzou@163.com

*Contributed equally

Key words: diabetic nephropathy, notoginsenoside R1, podocyte, $\mathrm{PI} 3 \mathrm{~K} /$ Akt signaling pathway as well as an anti-inflammatory cytokine (IL-10) were assessed, and the apoptosis of podocytes was quantified. Finally, the phosphoinositide 3-kinase (PI3K)/Akt signaling pathway and the involvement of nuclear factor $-\kappa \mathrm{B}(\mathrm{NF}-\kappa \mathrm{B})$ inactivation was further analyzed. In this study, NR1 improved renal function by ameliorating histological alterations, increasing the expression of nephrin and podocin, decreasing the expression of desmin, and inhibiting both the inflammatory response as well as the apoptosis of podocytes. Furthermore, NR1 treatment increased the phosphorylation of both PI3K (p85) and Akt, indicating that activation of the PI3K/Akt signaling pathway was involved. Moreover, NR1 treatment decreased the phosphorylation of $N F-\kappa B$ (p65), suggesting the downregulation of NF- $\kappa$ B. This is the first study to the best of our knowledge, to clearly demonstrate that NR1 treatment ameliorates podocyte injury by inhibiting both inflammation and apoptosis through the PI3K/Akt signaling pathway.

\section{Introduction}

Diabetic nephropathy (DN) is a major complication of diabetes which causes thickening of the glomerular basement membrane, glomerular hypertrophy and mesangial expansion, resulting in overt renal disease. The filtration barrier of the kidney is formed by the glomerulus, which is composed of a fenestrated endothelium, glomerular basement membrane, and podocytes that form slit diaphragms (1). When podocytes are damaged, chronic kidney disease (CKD) and end-stage renal disease (ESRD) occur. The terminally differentiated podocytes (also known as glomerular visceral epithelial cells) are a group of highly specialized cells, which are derived from mesenchymal cells and possess a limited capacity to proliferate (2). Podocytes have been recognized as critical regulators of glomerular injury and podocyte injury is clinically characterized by proteinuria and podocyte loss, which are observed as the apoptosis of podocytes. As podocytes are terminally differentiated cells, their loss in association with 
their inability to be replaced or regenerated, ultimately results in glomerulosclerosis $(2,3)$. Diabetic glomerular diseases remain among the major causes of CKD and ESRD (4). With the increasing prevalence of diabetes, DN and podocyte protection has drawn increasing attention. Despite extensive research into understanding and treating DN, investigating the application of herbal medicine in the treatment of DN as well as the underlying mechanisms, remains a challenging task.

Notoginsenoside R1 (NR1) is the major component of Fufang Xiancao Keli (a Chinese herbal medicine compound), which has been used clinically for the treatment of DN for centuries in China (6). Previous studies have demonstrated that NR1, a major component of Panax notoginseng, attenuated renal ischemia-reperfusion injury in rats (5). A study on the application of NR1 in a rat model of DN indicated that NR1 ameliorated the glucose-induced impairment of podocyte adhesive capacity and subsequent podocyte depopulation, partly through the upregulation of $\alpha 3 \beta 1$ integrin (6). Gui et al showed that in rats with streptozotocin (STZ)-induced diabetes, treatment with NR1 for 12 weeks partially restored the number of podocytes per glomerular volume and glomerular $\alpha 3 \beta 1$ integrin expression (6). However, further exploration of the mechanism responsible for NR1-induced podocyte protection is urgently needed.

To maintain podocyte integrity, nephrin, podocin and desmin have been demonstrated to play a pivotal role (7-9), and their expression levels were further analyzed in this study. Mounting evidence has suggested that there is a decrease in podocyte number in diabetic glomerular disease (10-13). Accompanying the decreased podocyte number, the consequences include proteinuria and glomerulosclerosis. Particularly in DN, studies have shown that proteinuria increased as the podocyte number decreased $(12,14)$. Thus, in the present study we aimed to examine the protective effects of NR1 in podocytes, initially through the measurement of proteinuria. In addition, emerging experimental and clinical literature suggests that apoptosis is a major cause of reduced podocyte numbers, which ultimately leads to proteinuria. Using terminal deoxynucleotidyl transferase-mediated deoxyuridin triphosphate nick end labeling (TUNEL) staining and quantifying the number of apoptotic cells per field, the protection effects of NR1 in podocytes were further assessed. As demonstrated by Schiffer $e t a l$, who were among the first to provide evidence for the increased apoptosis of podocytes in experiments performed in transgenic mice with elevated levels of tumor growth factor- $\beta 1$ (TGF- $\beta 1$ ) (15), the serum levels of TGF- $\beta 1$ and tumor necrosis factor- $\alpha$ (TNF- $\alpha$ ) were analyzed. In addition, an increasing body of experimental and clinical literature shows that the phosphoinositide 3-kinase (PI3K)/Akt signaling pathway is widely expressed in eukaryotes and plays essential roles in growth, differentiation, proliferation and survival (16). Furthermore, a study of puromycin aminonucleoside (PAN)-induced podocyte injury revealed that the tyrosine phosphorylation of nephrin and the nephrin-p85 interaction were reduced, which is indicative of decreased Akt activity in podocytes (17). Another study investigating the correlation between the PI3K/Akt signaling pathway and podocyte numbers showed that dexamethasone inhibited podocyte apoptosis by stabilizing the PI3K/Akt signaling pathway in vitro (18). Thus, in the present study, we aimed to further examine our hypothesis that NR1 ameliorates podocyte injury in rats with STZ-induced DN by inhibiting the apoptosis of podocytes through the PI3K/Akt signaling pathway.

\section{Materials and methods}

Chemicals. NR1 (chemical structure $\mathrm{C}_{47} \mathrm{H}_{80} \mathrm{O}_{18}$; molecular weight, $933 \mathrm{Da}$ ) was purchased from Sigma-Aldrich Chemicals (St. Louis, MO, USA), and the purity of NR1 was $>98 \%$. All other chemicals and reagents were also purchased from Sigma-Aldrich Chemicals. Podocin (\#SC-21009), nephrin (\#SC-19000), desmin (\#SC-14026), NF-kB (\#sc-109) and $\beta$-actin (\#sc-47778) antibodies were purchased from Santa Cruz Biotechnology (Santa Cruz, CA, USA). Phosphorylated (p-)PI3K-p85(Y458) (\#4228), PI3K-p85 (\#4257), p-Akt (S473) (\#4060), Akt (\#4685) and p-NF-kB p65 (\#3033) were purchased from Cell Signaling Technologies (Danvers, MA, USA). Goat anti-rabbit secondary antibody conjugated with HRP (\#11-035-003) and goat anti-mouse antibody conjugated with HRP (\#211-035-109) were purchased from Jackson ImmunoResearch Laboratories (West Grove, PA, USA).

Experimental design and animal groups. Six-week-old, healthy male Sprague-Dawley (SD) rats (200-220 g) were obtained from and kept at the Animal Center of Sichuan Provincial People's Hospital (Chengdu, China). The rats were randomly divided into the following five groups $(\mathrm{n}=10)$ : i) normal control $(\mathrm{NC})$ group of rats which received $0.1 \mathrm{~mol} / \mathrm{l}$ citrate buffer solution (control solution); ii) DN group of rats which received an intraperitoneal (i.p.) injection of STZ (Sigma-Aldrich Chemicals),

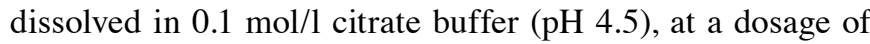
$65 \mathrm{mg} / \mathrm{kg}$; iii) rats with DN treated with a low dose of NR1 [5 mg/kg.day (6); DN + NR1 (low) group]; iv) DN rats treated with a medium dose of NR1 [10 mg/kg dday (6); DN + NR1 (medium) group]; and v) DN rats treated with a high dose of NR1 $[20 \mathrm{mg} / \mathrm{kg} \cdot$ day (5); DN + NR1 (high) dose group]. NR1 was administered orally.

For the DN group of rats, 3 days after the injection of STZ, their fasting blood glucose levels were measured using a One Touch Ultra Blood Glucose meter (LifeScan Inc., Milpitas, CA, USA). Animals with fasting blood glucose levels $>16.7 \mathrm{mmol} / \mathrm{l}$ were considered to be diabetic rats, and were used in the present study. NR1 was administered after the induction of diabetes, and the control group (DN group) was given an equivalent volume of vehicle. Every 4 weeks, fasting blood glucose levels and body weight were measured, and $24 \mathrm{~h}$ urine samples were also collected in metabolic cages, starting at 3 days after STZ injection. All animals were euthanized by $\mathrm{CO}_{2}$ inhalation at 16 weeks after the initiation of NR 1 or vehicle treatment. At the end of the experiment and prior to sacrifice, blood samples were obtained from the inferior vena cava, and the kidneys were harvested immediately. Animal handling was performed in accordance with the Ethics Committee of Sichuan Provincial People's Hospital, and all animals were kept under a $12 \mathrm{~h}$ light/dark cycle with free access to water and food.

Assessment of renal function. To assess renal function, blood urea nitrogen (BUN) and serum creatinine levels were deter- 
mined using an automatic biochemistry analyzer (Hitachi, Tokyo, Japan). Urinary albumin concentrations were measured using an enzyme-linked immunosorbent assay (ELISA) kit (Runyu Biotechnology Co., Shanghai, China), according to the manufacturer's instructions.

Histological analysis. The renal samples were fixed in $10 \%$ formalin for $24 \mathrm{~h}$ at room temperature, and histological slides were prepared according to the standardized protocol in our laboratory (19). The sections were then stained with periodic acid-Schiff (PAS) and Trichrome reagent (Masson kit) (both from Sigma-Aldrich Chemicals), and examined under a microscope (Nikon 80i microscope; Nikon, Tokyo, Japan) in a blinded manner. The extent of renal injury was estimated by the morphometric assessment of the degree of mesangial matrix expansion and of glomerular enlargement. A point-counting method was used to quantify mesangial matrix deposition, and 20 randomly selected non-overlapping PAS-stained glomeruli from each rat were analyzed (20).

Evaluation of apoptosis by the TUNEL assay. Paraffinembedded kidney tissues were cut into $4 \mu \mathrm{m}$ thick sections. A TUNEL assay was performed to assess DNA fragmentation. The assay was performed according to the manufacturer's instructions (Promega, Madison, W1, USA) as previously described (21). The total cell population and the number of TUNEL-positive cells were manually counted, and double checked using Image Pro Plus 6.3 software (Media Cybernetics, Silver Spring, MD, USA).

Measurement of cytokines. For measuring pro-inflammatory mediators [TNF- $\alpha$, TGF- $\beta 1$, interleukin (IL)- 1 and IL-6] and an anti-inflammatory mediator (IL-10) in the serum, blood samples were collected at the beginning and end of NR1 treatment. The serum cytokines were assayed using specific ELISA kits for rats according to the manufacturer's instructions (R\&D Systems, Minneapolis, MN, USA).

Western blot analysis. The proteins were lysed in $50 \mathrm{mM}$ Tris- $\mathrm{HCl}, \mathrm{pH} 8.0,150 \mathrm{mM} \mathrm{NaCl}, 5 \mathrm{mM}$ ethylenediaminetetraacetic acid (EDTA), 1\% NP-40, and protease inhibitor cocktail (22). The protein lysates were centrifuged at $13,400 \mathrm{x} g$ for $10 \mathrm{~min}$. Supernatants were collected and loaded on a NuPage Novex $10 \%$ Bis-Tris gel (Life Technologies, Carlsbad, CA, USA) for electrophoresis. Following electrophoresis, the proteins were transferred onto polyvinylidene fluoride membranes (Pall Corporation, Port Washington, NY, USA) in NuPage transfer buffer (Life Technologies). The membranes were blocked with $5 \%$ bovine serum albumin in TBST for $1 \mathrm{~h}$. The blots were incubated overnight at $4^{\circ} \mathrm{C}$ with primary antibodies at the dilutions recommended by the manufacturer, followed by incubation with a horseradish peroxidase (HRP)-conjugated secondary antibody. Chemiluminescence detection was performed using Immobilon Western Chemiluminescent HRP substrate (Millipore, Billerica, MA, USA), and measured directly by a BioSpectrum Imaging System (UVP, Upland, CA, USA). Equal protein loading was confirmed by immunostaining against $\beta$-actin. The signal intensity was analyzed by ImageQuant software (Molecular Dynamics, Sunnyvale, CA, USA) and normalized to $\beta$-actin.
Reverse transcription-quantitative polymerase chain reaction $(R T-q P C R)$. Total RNA was extracted from samples of isolated rat glomeruli using TRIzol reagent (Life Technologies). Reverse transcription was performed using a high capacity cDNA reverse transcription kit according to the manufacturer's instructions (Life Technologies). Two-step RT-qPCR analysis of the expression of nephrin, podocin and desmin was performed using Applied Biosystems Power SYBR ${ }^{\circledR}$-Green PCR master mix (Life Technologies) with 40 cycles of $45 \mathrm{sec}$ at $95^{\circ} \mathrm{C}$ and $1.5 \mathrm{~min}$ at $55^{\circ} \mathrm{C}$ on an Applied Biosystems 7900 Real-Time PCR system using primers as described previously (23-25). Fluorescence data were collected at $55^{\circ} \mathrm{C}$ after each cycle. After the final cycle, melting curve analysis of all samples was conducted within the range of $55-95^{\circ} \mathrm{C}$. Relative quantification of gene expression was performed using glyceraldehyde-3-phosphate dehydrogenase (GAPDH) as an internal control. The threshold cycle and $2^{-\Delta \Delta C t}$ method were used for calculating the relative amount of the target RNA (26). RT-qPCR always included a no-template sample as a negative control. The experiments were repeated twice, and each sample was tested in triplicate.

Statistical analysis. The statistical analyses were performed using GraphPad Prism (version 5.0 for windows) statistical software package (GraphPad Software, San Diego, CA, USA). All results are expressed as the means \pm standard error of mean (SEM) for each group. One-way analysis of variance (ANOVA) and the Mann-Whitney $U$ test were used to determine the level of significance among the groups, and $\mathrm{P}<0.05$ was considered to indicate a statistically significant difference.

\section{Results}

$N R 1$ treatment reduces proteinuria in rats with $D N$. To assess the renal protective effect of NR1, fasting blood glucose levels and body weights were measured every 4 weeks starting from 3 days after STZ injection. As shown in Table I, the rats in the DN group demonstrated elevated blood glucose levels, which were significantly higher than those of the non-diabetic rats $(\mathrm{P}<0.05)$. During the study period, a low dose of NR1 did not induce a decrease in blood glucose levels ( $\mathrm{P}>0.05)$, whereas with the elevated dosage of NR1, the blood glucose levels were significantly lower than those of the DN group $(\mathrm{P}<0.05)$, particularly from 12 weeks onwards. Moreover, the body weight of the NR1-treated (medium dose and high dose) rats increased steadily, compared with those of the rats in the DN group $(\mathrm{P}<0.05)$ (Table II). Furthermore, general parameters, namely BUN, serum creatinine, the kidney-to-body weight ratio and proteinuria were measured. With the progression of DN, proteinuria was increased in the diabetic rats $(\mathrm{P}<0.05)$ (Fig. 1A). As expected, the low dose NR1-treated rats did not show significantly decreased proteinuria levels ( $P>0.05)$, and neither did the medium dose NR1-treated rats. However, the proteinuria levels of rats treated with a high dose of NR1 were significantly lower than those of the rats in the DN group. Further evidence of NR1-induced podocyte protection was obtained from the BUN concentrations (Fig. 1B) and serum creatinine levels (Fig. 1C). As expected, the kidney-to-body weight ratios increased approximately 2-fold 
Table I. Effect of treatment on blood glucose levels in all experimental groups ( $\mathrm{mmol} / \mathrm{l}$, means $\pm \mathrm{SEM})$.

\begin{tabular}{|c|c|c|c|c|c|}
\hline Group & 0 week & 4 weeks & 8 weeks & 12 weeks & 16 weeks \\
\hline $\mathrm{NC}$ & $5.82 \pm 0.12$ & $5.56 \pm 0.26$ & $5.23 \pm 0.16$ & $5.44 \pm 0.09$ & $5.47 \pm 0.14$ \\
\hline DN & $25.13 \pm 2.22^{\mathrm{a}}$ & $26.48 \pm 1.57^{\mathrm{a}}$ & $27.11 \pm 3.28^{a}$ & $28.54 \pm 2.14^{\mathrm{a}}$ & $26.42 \pm 2.64^{\mathrm{a}}$ \\
\hline DN + NR1 (low) & $24.98 \pm 1.35$ & $25.73 \pm 2.33$ & $26.75 \pm 2.50$ & $25.38 \pm 1.79$ & $25.10 \pm 2.34$ \\
\hline $\mathrm{DN}+\mathrm{NR} 1$ (medium) & $25.06 \pm 2.16$ & $24.96 \pm 1.89$ & $23.67 \pm 1.84$ & $21.43 \pm 2.17^{\mathrm{b}}$ & $18.62 \pm 1.59^{c}$ \\
\hline DN + NR1 (high) & $24.92 \pm 1.05$ & $25.73 \pm 2.15$ & $21.58 \pm 1.49^{b}$ & $16.35 \pm 3.26^{\mathrm{c}}$ & $13.75 \pm 3.64^{\mathrm{c}}$ \\
\hline
\end{tabular}

$\mathrm{n}=10$ in each group, ${ }^{\mathrm{a}} \mathrm{P}<0.01$ vs. $\mathrm{NC}$ group at the same time point; ${ }^{\mathrm{b}} \mathrm{P}<0.05$ and ${ }^{\mathrm{c}} \mathrm{P}<0.01$ vs. DN group at the same time point. NC, normal control; DN, diabetic nephropathy; NR1, notoginsenoside R1.

Table II. Effect of treatment on body weight in all experimental groups (g, means \pm SEM).

\begin{tabular}{|c|c|c|c|c|c|}
\hline Group & 0 week & 4 weeks & 8 weeks & 12 weeks & 16 weeks \\
\hline $\mathrm{NC}$ & $214 \pm 26$ & $389 \pm 31$ & $459 \pm 26$ & $516 \pm 34$ & $547 \pm 48$ \\
\hline $\mathrm{DN}$ & $210 \pm 23$ & $218 \pm 17^{\mathrm{a}}$ & $215 \pm 18^{b}$ & $207 \pm 11^{\mathrm{b}}$ & $198 \pm 13^{b}$ \\
\hline DN + NR1 (low) & $218 \pm 13$ & $220 \pm 21$ & $218 \pm 15$ & $216 \pm 19$ & $219 \pm 25$ \\
\hline DN + NR1 (medium) & $216 \pm 21$ & $218 \pm 23$ & $225 \pm 19$ & $237 \pm 28^{c}$ & $239 \pm 36^{d}$ \\
\hline DN + NR1 (high) & $218 \pm 18$ & $229 \pm 17$ & $239 \pm 15^{c}$ & $245 \pm 15^{\mathrm{d}}$ & $258 \pm 19^{d}$ \\
\hline
\end{tabular}

$\mathrm{n}=10$ in each group, ${ }^{\mathrm{a}} \mathrm{P}<0.05$ and ${ }^{\mathrm{b}} \mathrm{P}<0.01$ vs. $\mathrm{NC}$ group at the same time point; ${ }^{\mathrm{c}} \mathrm{P}<0.05$ and ${ }^{\mathrm{d}} \mathrm{P}<0.01$ vs. DN group at the same time point. $\mathrm{NC}$, normal control; DN, diabetic nephropathy; NR1, notoginsenoside R1.

$\mathbf{A}$

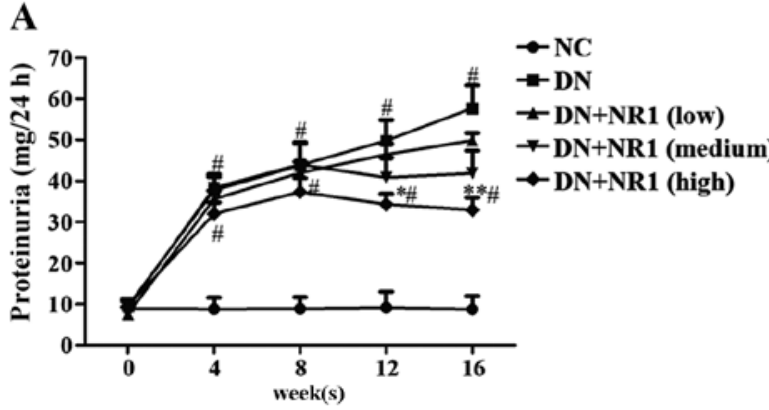

C

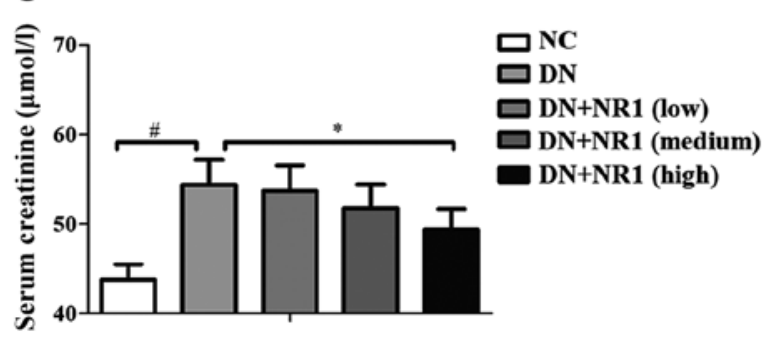

B

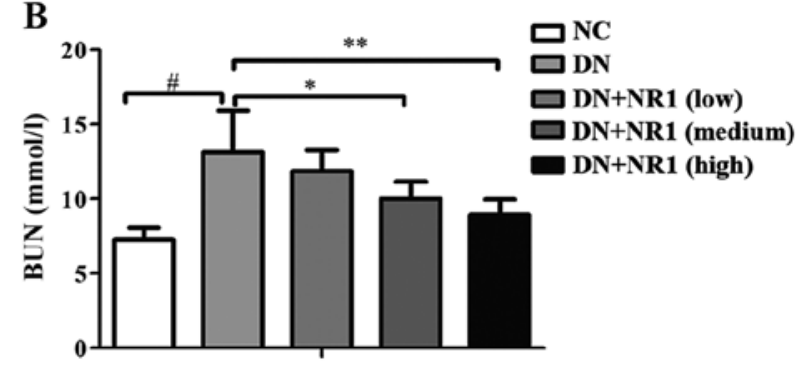

D

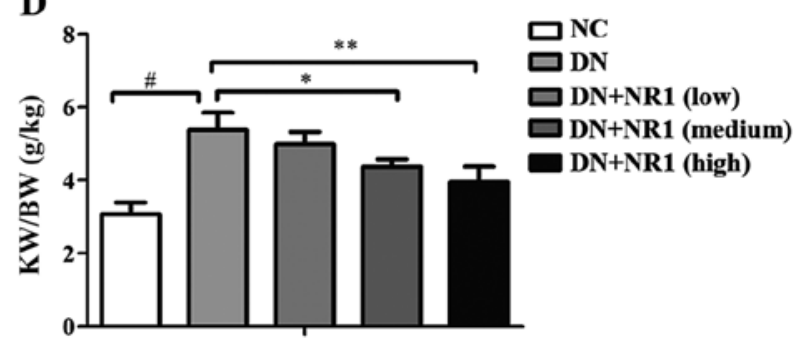

Figure 1. Assessment of the renal protective role of notoginsenoside R1 (NR1) using general parameters. (A) Time course study of proteinuria levels. " $\mathrm{P}<0.05$ and ${ }^{* *} \mathrm{P}<0.01$ vs. diabetic nephropathy $(\mathrm{DN})$ group at the same time point. ${ }^{*} \mathrm{P}<0.05$ vs. normal control (NC) group at the same time point. (B) Blood urea nitrogen (BUN) concentrations at week 16. (C) Serum creatinine levels at week 16. (D) Kidney-to-body weight ratio (KW/BW) at week 16. $\mathrm{n}=10 \mathrm{in}$ each group, ${ }^{*} \mathrm{P}<0.05$ and ${ }^{* *} \mathrm{P}<0.01$ vs. $\mathrm{DN}$ group; ${ }^{*} \mathrm{P}<0.01$ vs. NC group. Data are the means \pm SEM from at least 2 independent experiments.

in the rats of the DN group compared with those of the non-diabetic controls, whereas in those treated with NR1, those ratios were decreased significantly (Fig. 1D) $(\mathrm{P}<0.05$ for the medium and the high dose NR1-treated groups vs. the DN group). The above data suggest that NR1 treatment signifi- cantly reduced proteinuria and protected podocytes in the rats with experimentally-induced diabetes.

NR1 treatment ameliorates the diabetes-induced histological alterations in rat kidneys. To further analyze the efficacy of NR1 
A

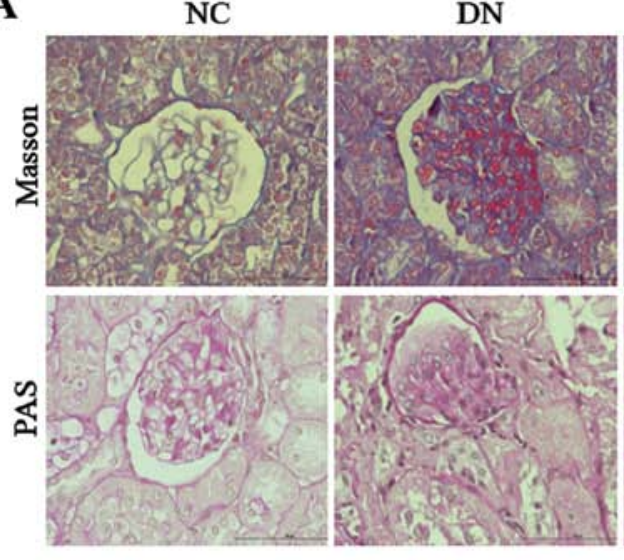

B

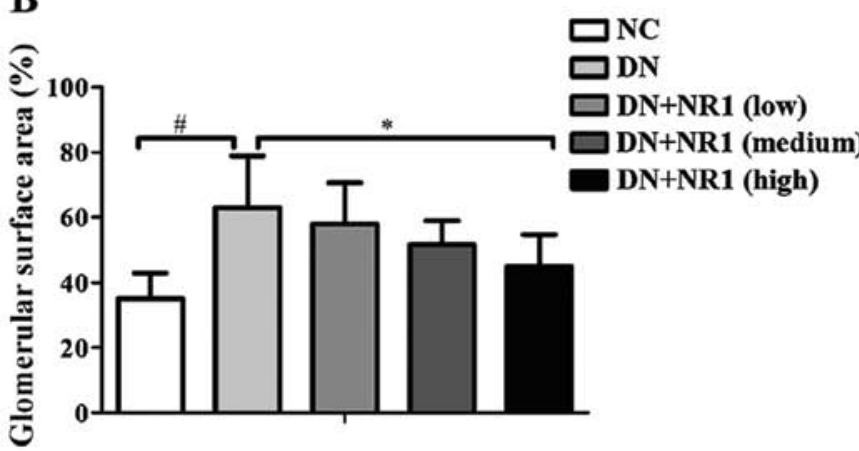

NR1 (low)

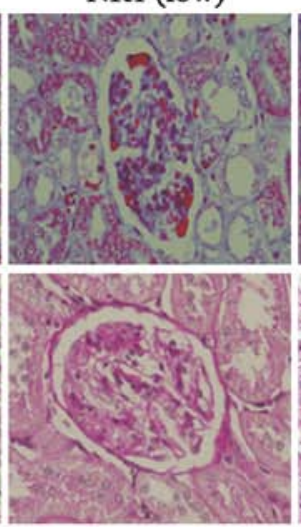

C

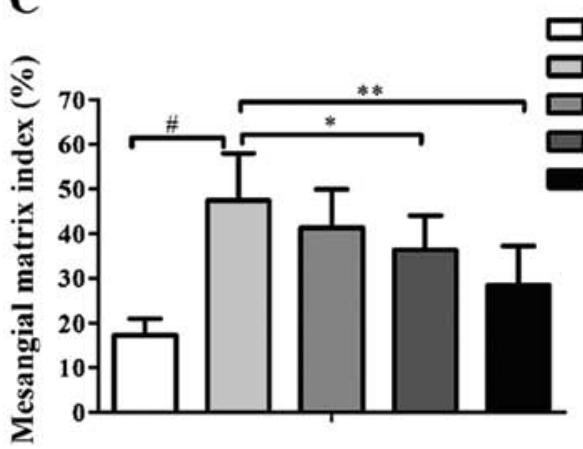

NR1 (high)

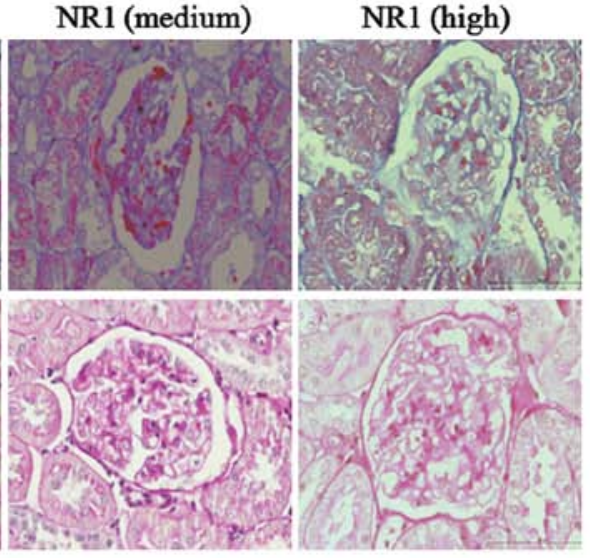

口NC

$\square \mathrm{DN}$

DN+NR1 (low)

DN+NR1 (medium)

DN+NR1 (high)

Figure 2. Notoginsenoside R1 (NR1) induces histological changes in rats with diabetic nephropathy (DN). (A) Representative images of Masson staining (upper panels) and periodic acid-Schiff (PAS) staining (lower panels) of kidney tissues from different groups (x400 magnification). (B) Glomerular surface area (percentage) of different groups. (C) Mesangial matrix index of different groups. ${ }^{*} \mathrm{P}<0.05$ and ${ }^{* *} \mathrm{P}<0.01$ vs. DN group; ${ }^{\#} \mathrm{P}<0.01$ vs. normal control (NC) group. $n=10$ in each group. Data are the means \pm SEM from 3 independent experiments.

in controlling DN in STZ-exposed rats, the histological changes in each group of rats were observed under a light microscope. As shown in Fig. 2A [upper panels, Masson staining; lower panels, PAS staining] as well as Fig. 2B and C, compared with the nondiabetic control group, the glomerular surface area increased 2 -fold and the glomerular mesangial matrix expanded significantly in the $\mathrm{DN}$ group ( $\mathrm{P}<0.05, \mathrm{DN}$ group vs. $\mathrm{NC}$ group). The quantitative histological analysis revealed that with the increased dosage of NR1, the percentage of the glomerular surface area decreased significantly (Fig. 2B) $(\mathrm{P}<0.05$, high dose NR1-treated group vs. DN group). Moreover, the expansion of the glomerular mesangial matrix in the NR1-treated groups (medium dose and high dose) decreased as well (Fig. 2C) $(\mathrm{P}<0.05$, medium and high dose NR1-treated groups vs. DN group). The above-mentioned histological evaluation confirmed that NR1 treatment ameliorates the structural changes in the diabetic kidney.

NRI induces changes in the expression levels of nephrin, podocin and desmin in the kidneys of rats with DN. Previous studies have demonstrated that nephrin and podocin are important slit diaphragm proteins in podocytes, therefore they are of vital importance in the maintenance of podocyte integrity (27). In the present study, in order to elucidate the mechanism responsible for the NR1-mediated improvement in the glomerular barrier function of rats with DN, we quantified the expression levels of nephrin and podocin using western blot analysis and RT-qPCR. Western blot analysis revealed a reduction in the expression of nephrin and podocin proteins in the rats with DN, whereas with the administration of increasing doses of NR1, the protein levels of nephrin and podocin were markedly restored (Fig. 3A). In addition, the mRNA levels of nephrin and podocin were in agreement with the protein expression levels, indicating that both nephrin and podocin were involved in NR1-medicated podocyte protection (Fig. 3B and C). Moreover, in order to elucidate the possibility that the protective effect of NR1 occurred through the maintenance of podocyte integrity, we examined the expression levels of desmin, a marker of podocyte injury. Judging from Fig. 3A and D, the expression level of desmin was hardly observed in the podocytes of non-diabetic rats; however, it increased by almost four times compared with that in the normal control $(\mathrm{P}<0.05)$ (Fig. 3D). As expected, NR1 treatment markedly decreased desmin expression levels, as shown by the results of western blot analysis (Fig. 3A) and RT-qPCR (Fig. 3D). These results indicated that NR1 protected against podocyte injury by increasing the expression of nephrin and podocin, and by decreasing the expression of desmin in rats with DN.

NR1 treatment inhibits the inflammatory response in rats with $D N$. In order to evaluate the possible involvement of NR1 in the regulation of inflammatory cytokines in DN rats, we further analyzed the secretion of inflammatory cytokines (TNF- $\alpha$, TGF- $\beta 1$, IL-1 and IL-6) and an anti-inflammatory cytokine (IL-10) in the serum. As shown in Fig. 4, the secretion of TNF- $\alpha$ (Fig. 4A), TGF- $\beta 1$ (Fig. 4B), IL-1 (Fig. 4C) and IL-6 (Fig. 4D) was increased in the serum of rats in the DN group, compared with the $\mathrm{NC}$ groups, and the secretion of 
A
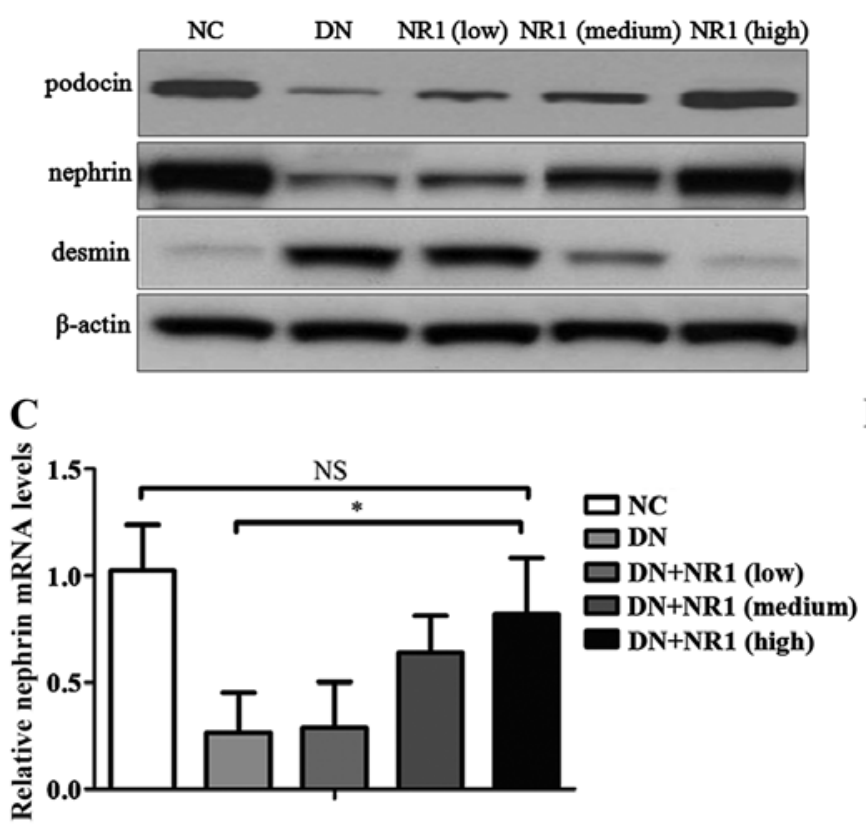

B
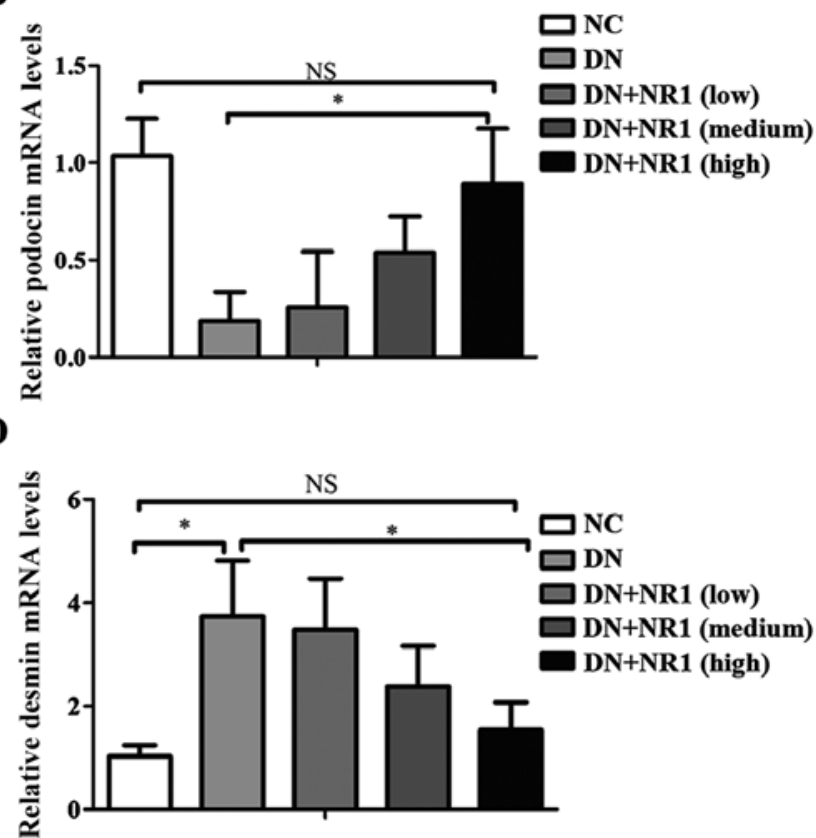

Figure 3. Notoginsenoside R1 (NR1) restores the expression of slit diaphragm proteins. (A) Representative western blots of podocin, nephrin, desmin and $\beta$-actin proteins isolated from the kidneys from different groups. Protein bands are representative of $>3$ independent experiments with similar results. (B-D) RT-qPCR of podocin, nephrin and desmin mRNA levels. ( ${ }^{*} \mathrm{P}<0.05$; NS, not significant). Data are the means \pm SEM from 3 independent experiments. NC, normal control; DN, diabetic nephropathy.

IL-10 (Fig. 4E) was decreased in the serum of rats with DN compared with the non-diabetic rats. However, following treatment with NR1, the levels of the inflammatory cytokines were significantly suppressed by NR1, whereas IL-10 levels were elevated. Taken together, our data indicated that NR1 treatment significantly suppressed the secretion of inflammatory cytokines and increased the secretion of an anti-inflammatory cytokine in rats with DN.

NR1 treatment ameliorates the diabetes-induced apoptosis of podocytes. To determine whether the protective effect of NR1 in podocytes was due to the amelioration of apoptosis, we performed TUNEL staining in the kidneys of the experimental rats. As shown in Fig. 5A, no positive signals were present in the glomeruli of the normal control rats and the high dose NR1-treated rats (Fig. 5A). In addition, there were significantly reduced numbers of TUNEL-positive cells in the kidneys of low and medium dose NR1-treated rats. The percentages of TUNEL-positive cells indicated that NR1 treatment decreased the diabetes-associated apoptosis of renal cells as compared with the diabetic control (Fig. 5B). Thus, these results suggest that NR1 treatment contributed to the podocyte protection by inhibiting the apoptosis of podocytes under our experimental conditions.

NR1 treatment induces activation of the PI3K/Akt signaling pathway in rats with DN. In order to explore the mechanisms responsible for the NR1-mediated inhibition of apoptosis and the suppressed inflammatory response in rats with DN, we examined whether the PI3K/Akt signaling pathway is suppressed in the podocytes of STZ-exposed rats and whether NR1 is capable of reversing these changes. Western blot analysis of p-PI3K-p85 (Tyr458), total PI3K-p85, p-Akt
(Ser473) and total Akt was performed, and it indicated that the phosphorylation of both PI3K-p85 and Akt was attenuated in the DN groups, compared with that of the NC control group (Fig. 6A). Further quantification of the grey scale of each blot was performed and the ratio of p-p85 vs. total PI3K-p85 protein was calculated. As shown in Fig. 6B, NR1 gradually increased the phosphorylation of $\mathrm{p} 85$, in a dose-dependent manner. Furthermore, the enhanced phosphorylation of Akt was found in the NR1-treated rats with $\mathrm{DN}(\mathrm{P}<0.05$, compared with DN group), whereas the expression level of $\mathrm{p}$-Akt in the rats with DN was significantly suppressed $(\mathrm{P}<0.05$, compared with NC group) (Fig. 6C). The phosphorylation data of both p85 and Akt demonstrated that the PI3K/Akt signaling pathway was suppressed in the rats with DN, whereas NR1 protected podocytes and improved proteinuria by activating the PI3K/Akt signaling pathway.

NR1 treatment inhibits nuclear factor $-\kappa B(N F-\kappa B)$ p65 phosphorylation in rats with $D N$. Mounting evidence suggests that diabetes activates $\mathrm{NF}-\kappa \mathrm{B}$ signaling, which regulates the expression of several genes involved in the inflammatory response (28). Thus, to further evaluate the mechanism of NR1-induced podocyte protection, western blot analysis was employed to assess NF- $\kappa \mathrm{B}$ activation by evaluating the expression of total p65 and the extent of p65 activation. In the kidneys of rats with STZ-induced diabetes, increased phosphorylation of p65 was observed, compared with the non-diabetic rats (Fig. 6A). However, following treatment with NR1, p65 phosphorylation was significantly decreased (Fig. 6D), indicating the inactivation of $\mathrm{NF}-\kappa \mathrm{B}$. Taken together, these findings suggest that NR1 induced podocyte protection, apoptosis inhibition, and inflammation suppression by activating the PI3K/Akt signaling pathway and downregulating NF- $\mathrm{B}$. 
A
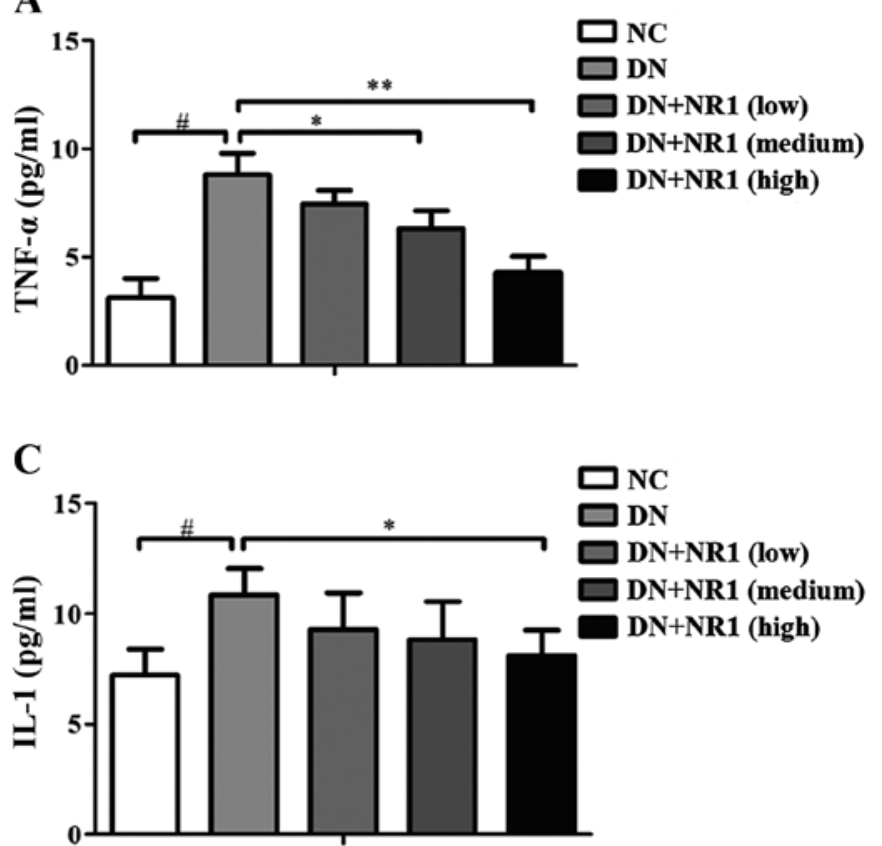

B

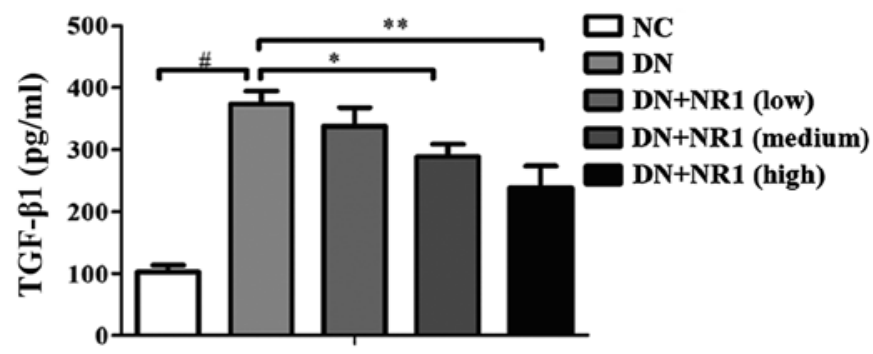

D

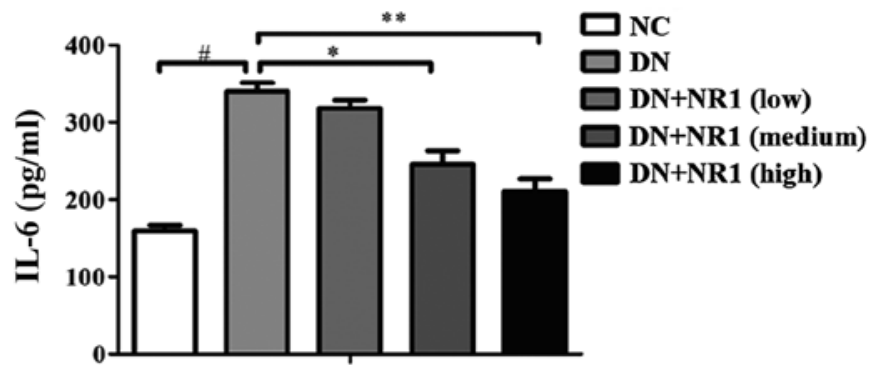

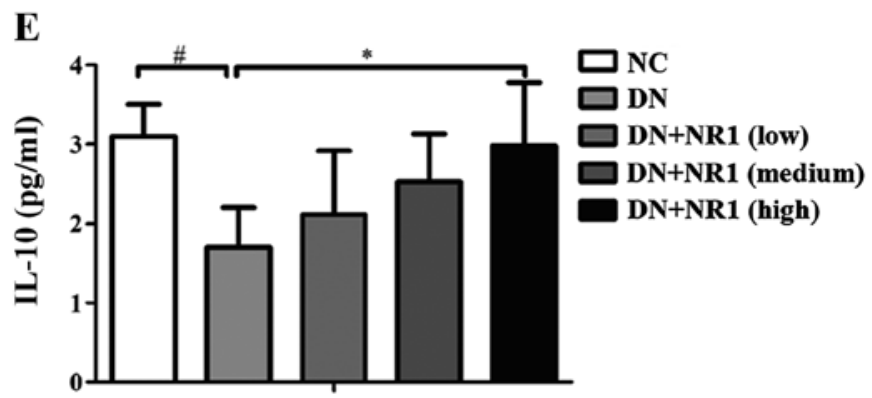

Figure 4. Notoginsenoside R1 (NR1) decreases the secretion of inflammatory cytokines and increases the secretion of an anti-inflammatory cytokine in rats with diabetic nephropathy (DN). Comparison of the serum levels of (A) tumor necrosis factor (TNF)- $\alpha$, (B) tumor growth factor (TGF)- $\beta 1$, (C) interleukin (IL)-1, (D) IL-6 and (E) IL-10 in different groups. Data are the means \pm SEM from 3 independent experiments. "P<0.05 and ** $\mathrm{P}<0.01$ vs. DN group; ${ }^{\#} \mathrm{P}<0.01$ vs. normal control (NC) group.

\section{Discussion}

According to epidemiologic observations, the prevalence of diabetes has increased significantly in recent decades all over the world (29). Of all diabetic complications, DN has drawn special focus, as the incidence of end-stage renal failure in diabetic patients has markedly increased in recent years (30). The high prevalence of the disease calls for intense research into ways of protecting podocytes, which have been recognized as critical regulators of glomerular injury (14). As the available treatment options for glomerular injury are limited to alkylating agents, steroids, angiotensin-converting enzyme inhibitors and angiotensin receptor blockers, exploring herbal medicine has attracted particular attention (31). A study by Gui et al (6) demonstrated that NR1 was involved in the upregulation of $\alpha 3 \beta 1$ integrin expression and the inhibition of oxidative stress; however, the mechanism responsible for NR1-induced podocyte protection remains to be elucidated. In the present study, NR1 treatment significantly decreased proteinuria, plasma and urinary biomarkers of renal function as well as blood glucose levels. The plasma and urinary biochemical results indicate that NR1 ameliorates the diabetes-associated compromised renal function. Furthermore, histological evaluation of the rat kidneys confirmed that NR1 significantly ameliorated diabetes-induced structural damage in a dose-dependent manner. More importantly, this is the first study, to the best of our knowledge, to delineate the protective role of NR1 against podocyte injury by activating the PI3K/Akt signaling pathway in rats with DN.

Considerable evidence indicates that nephrin is a podocyte protein crucial for the inter-podocyte slit membrane structure and the maintenance of an intact filtration barrier (32). It has been demonstrated that the modulation of nephrin expression contributes to the loss of glomerular filtration function, and is associated with the extent of proteinuria in DN (33). Similarly, the injection of anti-nephrin antibody in animals induced substructural alterations of the slit diaphragm with reductions in permselectivity and consequently proteinuria (34). In addition, the inactivation of the nephrin gene in mice by homologous recombination resulted in severe proteinuria, and partial foot process effacement (35). Thus, therapies targeted at correcting podocyte nephrin may be of value in the management of diabetes (36). It was reported that podocin is a member of the stomatin family, and it consists of hairpin-like integral 

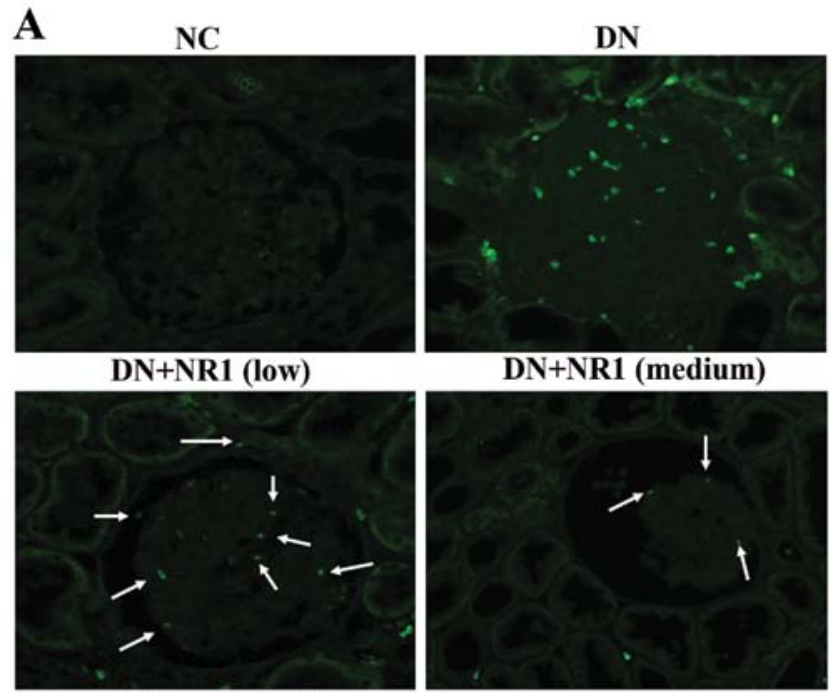

DN+NR1 (medium)

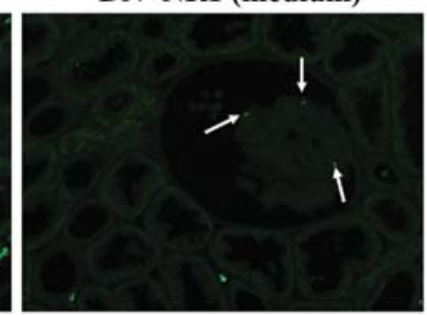

DN+NR1 (high)
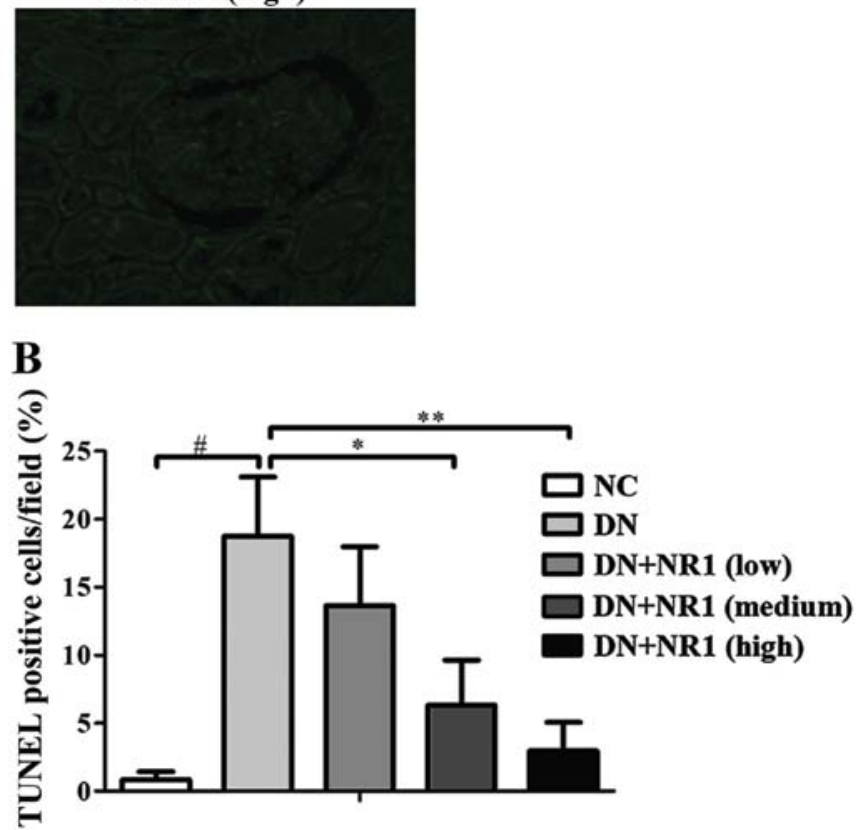

Figure 5. Notoginsenoside R1 (NR1) inhibits the apoptosis of podocytes. (A) Representative images of immunofluorescent staining of TUNEL (green)-labeled apoptotic cells from the different groups. White arrows point to the TUNEL-labeled cells. (B) Percentage of TUNEL-positive cells in the different groups. At least 20 randomly selected non-overlapping TUNELstained glomeruli from each rat were analyzed. $n=8$ for each group. The graph shows the means \pm SEM from at least 2 independent experiments ${ }^{*} \mathrm{P}<0.05$ and ${ }^{* *} \mathrm{P}<0.01$ vs. diabetic nephropathy $(\mathrm{DN})$ group; ${ }^{*} \mathrm{P}<0.01$ vs. normal control (NC) group.

membrane proteins. Further studies have demonstrated that podocin is expressed in glomerular podocytes, and serves in the structural organization of the slit diaphragm and the regulation of its filtration function $(37,38)$. Thus, podocin serves as a scaffolding molecule to localize nephrin, and the disruption of slit diaphragm proteins such as nephrin and podocin plays a significant role in the development and progression of DN. In the present study, we observed that in rats with STZ-induced DN, the expression of nephrin and podocin was decreased significantly, which was indicative of the disruption of the slit diaphragm proteins. However, when the rats with DN were treated with NR1, the major component of $P$. notoginseng, the expression of both nephrin and podocin was enhanced, which indicated that NR1 induced podocyte protection by regulating the expression of nephrin and podocin. In addition to nephrin and podocin, desmin, an intermediate filament protein, is also regulated in response to podocyte injury. Zou et al showed that the upregulation of desmin may increase the mechanical stability of cells, thus enabling podocytes to undergo morphological changes on the tensile glomerular capillary wall (39). Our results indicated that the expression level of desmin increased significantly in the rats with DN, and NR1 treatment markedly decreased desmin expression. These results suggest that NR1 protects podocytes from injury, by affecting the expression of slit diaphragm proteins in the rats with DN.

Mounting evidence, ranging from in vitro experiments to pathological examinations, have highlighted that inflammation plays a significant role in the development of DN $(40,41)$. Thus, the inhibition of inflammatory processes is one of the cardinal mechanisms of NR1-induced podocyte protection in DN. It has been reported that the renal expression of IL-1 is increased in animal models of DN (42). Moreover, researchers have demonstrated the strong association between mesangial expansion and the mRNA expression of IL- 6 in podocytes, indicating that IL-6 affects the extracellular matrix dynamics of podocytes (43). A clinical study of IL-6 serum levels demonstrated that IL-6 secretion was substantially higher in patients with DN (44). Thus, the expression of IL-1 and IL-6 was elevated in these models of DN, which is in accordance with our results. Additionally, our results demonstrated that the rats with DN receiving NR1 therapy showed decreased serum levels of these two cytokines, indicating that NR1 exerts anti-inflammatory effects. TNF- $\alpha$ is a cytokine with prominent inflammatory effects, and it is thought to play a pivotal role in the pathogenesis of DN. It has been reported that TNF- $\alpha$ levels are implicated in the development of renal hypertrophy and hyperfunction during the initial stage of DN (45). Accumulating clinical studies have revealed that the serum and urinary concentrations of TNF- $\alpha$ in patients with DN are substantially higher than in non-diabetic individuals (46). As expected, in the present study, NR1-treated rats with DN exhibited decreased serum TNF- $\alpha$ levels compared with the rats with DN treated with vehicle. Moreover, it has been reported that accumulated TGF- $\beta 1$ appears to play a pivotal role in the process of DN (47); apoptosis is increased in TGF- $\beta$ transgenic mice, which leads to a decrease in podocyte number and glomerulosclerosis. Follow-up studies showed that TGF- $\beta 1$-induced apoptosis of podocytes was mediated by specific Smad pathways $(15,48)$. Thus, evaluating TGF- $\beta 1$ secretion levels in the rats with $\mathrm{DN}$ and the NR1-treated rats with DN is of vital importance. In this study, TGF- $\beta 1$ secretion levels were enhanced significantly in the rats with DN, compared with those of the normal control rats, and with the increased dosage of NR1, the secretion levels of TGF- $\beta 1$ decreased significantly. On the other hand, IL-10 has been reported to block inflammation and improves renal function in a model of chronic renal disease (49). We observed elevated serum IL-10 secretion in the NR1-treated rats with DN. Taken together, the findings of the present study suggest that NR1 exerted protective effects in the podocytes of rats with DN by regulating the secretion of inflammatory cytokines as well as an anti-inflammatory cytokine. 

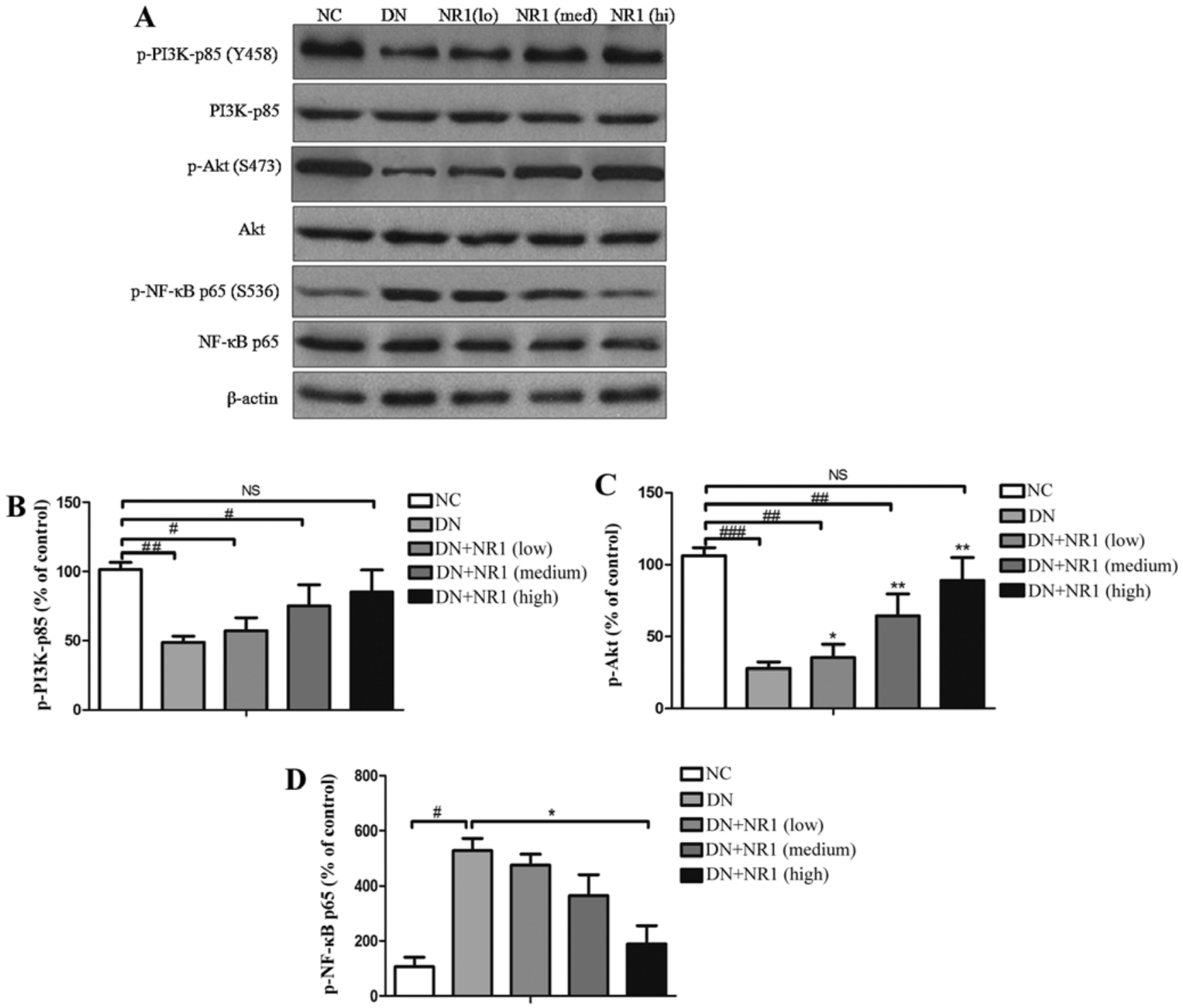

Figure 6. Notoginsenoside R1 (NR1) inhibits the apoptosis of podocytes and the inflammatory response through the PI3K/Akt pathway. (A) Representative western

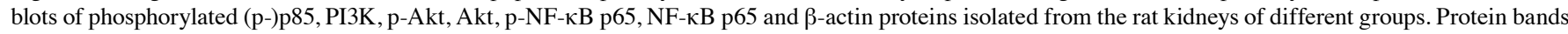
shown are representative of $>3$ independent experiments with similar results. (B and C) Percentage of phosphorylated protein with the corresponding control protein. All graphs show the means \pm SEM from at least 2 independent experiments. ${ }^{\#} \mathrm{P}<0.05$, ${ }^{\# \#} \mathrm{P}<0.01$ and ${ }^{\# \# \#} \mathrm{P}<0.01$ vs. normal control (NC) group. ${ }^{*} \mathrm{P}<0.05$

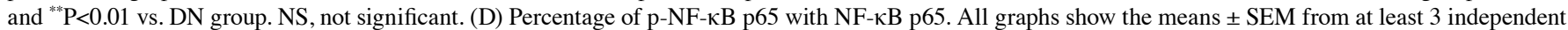
experiments. ${ }^{\mathrm{P}}<0.05$ vs. $\mathrm{DN}$ group; ${ }^{*} \mathrm{P}<0.01$ vs. $\mathrm{NC}$ group. $\mathrm{DN}$, diabetic nephropathy.

More importantly, the results suggest that NR1 exerts protective effects in DN by inhibiting apoptosis, activating the PI3K/Akt signaling pathway, and downregulating NF- $\mathrm{kB}$ expression. Many lines of evidence have shown that hyperglycemia directly induces apoptosis in cultured podocytes, thereby providing an additional possible explanation for the reduced podocyte numbers in DN (50). It has also been reported that a decrease in the podocyte number significantly correlated with reduced renal function and global glomerulosclerosis in diabetic patients $(12,14)$. Thus, our observation regarding the apoptosis of podocytes in the rats with $\mathrm{DN}$ was consistent with these previous findings. Furthermore, NR1 treatment decreased -apoptosis and restored the podocyte number as revealed by TUNEL staining and quantitative analysis of TUNEL-positive cell numbers, which indicated that NR1 treatment contributed to the maintenance of the podocyte number. To further explore the mechanism responsible for NR1-induced inhibition of apoptosis, the PI3K/Akt signaling pathway and the activation of NF- $\kappa \mathrm{B}$ was explored. Previous findings have demonstrated that PI3K was involved in nephrin-mediated actin reorganization in podocytes, and that disturbing nephrin-PI3K interactions may contribute to abnormal podocyte morphology and proteinuria (17). In this study, we observed the decreased expression of nephrin in the rats with DN, and NR1 treatment rescued those rats from DN by restoring the expression of nephrin. Furthermore, it has been reported that Akt kinase is activated by TGF- $\beta$ in diabetic kidneys, and TGF- $\beta$ may increase FoxO3a phosphorylation and transcriptional inactivation through PI3K/Akt, which also suggests the involvement of the PI3K/ Akt signaling pathway in the progression of DN $(51,52)$. Huber et al revealed that nephrin and CD2AP interacted 
with the p85 regulatory subunit of PI3K in vivo, recruited PI3K to the plasma membrane, and, together with podocin, stimulated PI3K-dependent Akt signaling in podocytes (53). Thus, we hypothesized that the PI3K/Akt signaling pathway may be involved. In this study, we observed a significant decrease in the phosphorylation of PI3K (p85) and Akt in the rats with STZ-induced DN. However, this inactivation of the PI3K/Akt signaling pathway was abrogated by NR1 administration. To further prove our hypothesis, our next study aims to provide in vitro evidence of NR1-induced $\mathrm{PI} 3 \mathrm{~K} / \mathrm{Akt}$ signaling pathway activation. As NF- $\mathrm{KB}$ regulates the expression of numerous genes that play key roles in the inflammatory response during human and experimental kidney injury (54), we postulated that the inactivation of NF- $\kappa \mathrm{B}$ was also involved in NR1-induced podocyte protection. In the present study, NR1 treatment significantly decreased the diabetes-induced activation of NF- $\mathrm{KB}$ by the phosphorylation of NF- $\kappa B$ at serine 536, whereas the expression of total NF- $\mathrm{kB}$ was unchanged in the different groups. Collectively, these findings suggest that NR1 treatment protects against diabetes-associated renal damage through the suppression of apoptosis, the inactivation of NF- $\mathrm{\kappa B}$ and the induction of PI3K/Akt activation.

In conclusion, to the best of our knowledge, this is the first study to examine the protective effects of NR1 treatment on podocytes which occur through the regulation of the PI3K/Akt and the NF- $\kappa B$ signaling pathway. The present study clearly demonstrated that NR1 ameliorates podocyte and renal injuries as well as apoptosis and inflammation by activating the $\mathrm{PI} 3 \mathrm{~K} / \mathrm{Akt}$ signaling pathway and inactivating NF- $\mathrm{KB}$. The present results provide new evidence for the further potential applications of NR1 in maintaining podocyte numbers and glomerular filtration barrier integrity as well as preventing proteinuria. However, further in vitro studies of the molecular mechanism responsible for the NR1-mediated protective effects on podocytes are warranted prior to conducting clinical investigations.

\section{Acknowledgements}

The present study was funded by the National Science Foundation of China (no. 81160434), the Guangxi Science Foundation (no. 2013GXNSFDA019016), and the Sichuan Science Foundation (no. 2015JY0183).

\section{References}

1. Wiggins RC: The spectrum of podocytopathies: a unifying view of glomerular diseases. Kidney Int 71: 1205-1214, 2007.

2. Shankland SJ: The podocyte's response to injury: role in proteinuria and glomerulosclerosis. Kidney Int 69: 2131-2147, 2006.

3. Mundel P and Shankland SJ: Podocyte biology and response to injury. J Am Soc Nephrol 13: 3005-3015, 2002.

4. Kumar PA, Brosius FC III and Menon RK: The glomerular podocyte as a target of growth hormone action: implications for the pathogenesis of diabetic nephropathy. Curr Diabetes Rev 7: 50-55, 2011.

5. Liu WJ, Tang HT, Jia YT, Ma B, Fu JF, Wang Y, Lv KY and Xia ZF: Notoginsenoside R1 attenuates renal ischemia-reperfusion injury in rats. Shock 34: 314-320, 2010.

6. Gui D, Wei L, Jian G, Guo Y, Yang J and Wang N: Notoginsenoside R1 ameliorates podocyte adhesion under diabetic condition through alpha3betal integrin upregulation in vitro and in vivo: Cell Physiol Biochem 34: 1849-1862, 2014.
7. Tryggvason K: Unraveling the mechanisms of glomerular ultrafiltration: nephrin, a key component of the slit diaphragm. J Am Soc Nephrol 10: 2440-2445, 1999.

8. Kestilä M, Lenkkeri U, Männikkö M, Lamerdin J, McCready P, Putaala H, Ruotsalainen V, Morita T, Nissinen M, Herva R, et al: Positionally cloned gene for a novel glomerular protein -nephrin is mutated in congenital nephrotic syndrome. Mol Cell 1: 575-582, 1998.

9. Khoshnoodi J, Sigmundsson K, Ofverstedt LG, Skoglund U, Obrink B, Wartiovaara J and Tryggvason K: Nephrin promotes cell-cell adhesion through homophilic interactions. Am J Pathol 163: 2337-2346, 2003.

10. McLaughlin JK, Lipworth L, Chow WH and Blot WJ: Analgesic use and chronic renal failure: a critical review of the epidemiologic literature. Kidney Int 54: 679-686, 1998.

11. Lemley KV, Lafayette RA, Safai M, Derby G, Blouch K, Squarer A and Myers BD: Podocytopenia and disease severity in IgA nephropathy. Kidney Int 61: 1475-1485, 2002.

12. Steffes MW, Schmidt D, McCrery R, Basgen JM; International Diabetic Nephropathy Study Group: Glomerular cell number in normal subjects and in type 1 diabetic patients. Kidney Int 59: 2104-2113, 2001

13. White KE and Bilous RW; Diabiopsies Study Group: Structural alterations to the podocyte are related to proteinuria in type 2 diabetic patients. Nephrol Dial Transplant 19: 1437-1440, 2004.

14. Pagtalunan ME, Miller PL, Jumping-Eagle S, Nelson RG, Myers BD, Rennke HG, Coplon NS, Sun L and Meyer TW: Podocyte loss and progressive glomerular injury in type II diabetes. J Clin Invest 99: 342-348, 1997.

15. Schiffer M, Bitzer M, Roberts IS, Kopp JB, ten Dijke P, Mundel P and Böttinger EP: Apoptosis in podocytes induced by TGF-beta and Smad7. J Clin Invest 108: 807-816, 2001.

16. Cantley LC: The phosphoinositide 3-kinase pathway. Science 296: 1655-1657, 2002.

17. Zhu J, Sun N, Aoudjit L, Li H, Kawachi H, Lemay S and Takano T: Nephrin mediates actin reorganization via phosphoinositide 3-kinase in podocytes. Kidney Int 73: 556-566, 2008.

18. Yu S and Li Y: Dexamethasone inhibits podocyte apoptosis by stabilizing the PI3K/Akt signal pathway. BioMed Res Int 2013: 326986, 2013.

19. Wang Y, Liu Y, Wang H, Li C, Qi P and Bao J: Agaricus bisporus lectins mediate islet $\beta$-cell proliferation through regulation of cell cycle proteins. Exp Biol Med (Maywood) 237: 287-296, 2012.

20. Sugimoto H, LeBleu VS, Bosukonda D, Keck P, Taduri G, Bechtel W, Okada H, Carlson W Jr, Bey P, Rusckowski M, et al: Activin-like kinase 3 is important for kidney regeneration and reversal of fibrosis. Nat Med 18: 396-404, 2012.

21. Wang Y, Wang H, Liu Y, Li C, Qi P and Bao J: Antihyperglycemic effect of ginsenoside $\mathrm{Rh} 2$ by inducing islet beta-cell regeneration in mice. Horm Metab Res 44: 33-40, 2012.

22. Jiagang D, Li C, Wang H, Hao E, Du Z, Bao C, Lv J and Wang Y: Amygdalin mediates relieved atherosclerosis in apolipoprotein $\mathrm{E}$ deficient mice through the induction of regulatory $\mathrm{T}$ cells. Biochem Biophys Res Commun 411: 523-529, 2011.

23. Rantanen M, Palmén T, Pätäri A, Ahola H, Lehtonen S, Aström E, Floss T, Vauti F, Wurst W, Ruiz P, et al: Nephrin TRAP mice lack slit diaphragms and show fibrotic glomeruli and cystic tubular lesions. J Am Soc Nephrol 13: 1586-1594, 2002.

24. Kawachi H, Koike H, Kurihara H, Sakai T and Shimizu F: Cloning of rat homologue of podocin: expression in proteinuric states and in developing glomeruli. J Am Soc Nephrol 14: 46-56, 2003.

25. Wiggins JE, Goyal M, Sanden SK, Wharram BL, Shedden KA, Misek DE, Kuick RD and Wiggins RC: Podocyte hypertrophy, 'adaptation' and 'decompensation' associated with glomerular enlargement and glomerulosclerosis in the aging rat: prevention by calorie restriction. J Am Soc Nephrol 16: 2953-2966, 2005.

26. Schmittgen TD and Livak KJ: Analyzing real-time PCR data by the comparative C(T) method. Nat Protoc 3: 1101-1108, 2008.

27. Huber TB and Benzing T: The slit diaphragm: a signaling platform to regulate podocyte function. Curr Opin Nephrol Hypertens 14: 211-216, 2005.

28. Li Q and Verma IM: NF-kappaB regulation in the immune system. Nat Rev Immunol 2: 725-734, 2002.

29. Whiting DR, Guariguata L, Weil C and Shaw J: IDF diabetes atlas: global estimates of the prevalence of diabetes for 2011 and 2030. Diabetes Res Clin Pract 94: 311-321, 2011.

30. Ritz E and Stefanski A: Diabetic nephropathy in type II diabetes. Am J Kidney Dis 27: 167-194, 1996. 
31. de Jong PE and Brenner BM: From secondary to primary prevention of progressive renal disease: the case for screening for albuminuria. Kidney Int 66: 2109-2118, 2004.

32. Aaltonen P, Luimula P, Aström E, Palmen T, Grönholm T, Palojoki E, Jaakkola I, Ahola H, Tikkanen I and Holthöfer H: Changes in the expression of nephrin gene and protein in experimental diabetic nephropathy. Lab Invest 81: 1185-1190, 2001.

33. Langham RG, Kelly DJ, Cox AJ, Thomson NM, Holthöfer H, Zaoui P, Pinel N, Cordonnier DJ and Gilbert RE: Proteinuria and the expression of the podocyte slit diaphragm protein, nephrin, in diabetic nephropathy: effects of angiotensin converting enzyme inhibition. Diabetologia 45: 1572-1576, 2002.

34. Orikasa M, Matsui K, Oite T and Shimizu F: Massive proteinuria induced in rats by a single intravenous injection of a monoclonal antibody. J Immunol 141: 807-814, 1988.

35. Putaala $H$, Soininen $R$, Kilpeläinen $P$, Wartiovaara $J$ and Tryggvason K: The murine nephrin gene is specifically expressed in kidney, brain and pancreas: inactivation of the gene leads to massive proteinuria and neonatal death. Hum Mol Genet 10: 1-8, 2001.

36. Benigni A, Gagliardini E, Tomasoni S, Abbate M, Ruggenenti P, KalluriR and Remuzzi G: Selective impairment of gene expression and assembly of nephrin in human diabetic nephropathy. Kidney Int 65: 2193-2200, 2004.

37. Schwarz K, Simons M, Reiser J, Saleem MA, Faul C, Kriz W, Shaw AS, Holzman LB and Mundel P: Podocin, a raft-associated component of the glomerular slit diaphragm, interacts with CD2AP and nephrin. J Clin Invest 108: 1621-1629, 2001.

38. Roselli S, Gribouval O, Boute N, Sich M, Benessy F, Attié T, Gubler MC and Antignac C: Podocin localizes in the kidney to the slit diaphragm area. Am J Pathol 160: 131-139, 2002.

39. Zou J, Yaoita E, Watanabe Y, Yoshida Y, Nameta M, Li H, Qu Z, Yamamoto T: Upregulation of nestin, vimentin, and desmin in rat podocytes in response to injury. Virchows Arch 448: 485-492, 2006.

40. Navarro-González JF and Mora-Fernández C: The role of inflammatory cytokines in diabetic nephropathy. J Am Soc Nephrol 19: 433-442, 2008.

41. Navarro-González JF, Mora-Fernández C, Muros de Fuentes M and García-Pérez J: Inflammatory molecules and pathways in the pathogenesis of diabetic nephropathy. Nat Rev Nephrol 7: 327-340, 2011.

42. Sassy-Prigent C, Heudes D, Mandet C, Bélair MF, Michel O, Perdereau B, Bariéty J and Bruneval P: Early glomerular macrophage recruitment in streptozotocin-induced diabetic rats. Diabetes 49: 466-475, 2000.
43. Suzuki D, Miyazaki M, Naka R, Koji T, Yagame M, Jinde K, Endoh M, Nomoto Y and Sakai H: In situ hybridization of interleukin 6 in diabetic nephropathy. Diabetes 44: 1233-1238, 1995.

44. Sekizuka K, Tomino Y, Sei C, Kurusu A, Tashiro K, Yamaguchi Y, Kodera S, Hishiki T, Shirato I and Koide H: Detection of serum IL-6 in patients with diabetic nephropathy. Nephron 68: 284-285, 1994.

45. DiPetrillo K, Coutermarsh B and Gesek FA: Urinary tumor necrosis factor contributes to sodium retention and renal hypertrophy during diabetes. Am J Physiol Renal Physiol 284: F113-F121, 2003.

46. Navarro JF and Mora-Fernández C: The role of TNF-alpha in diabetic nephropathy: pathogenic and therapeutic implications. Cytokine Growth Factor Rev 17: 441-450, 2006.

47. Mizuno S and Nakamura T: Suppressions of chronic glomerular injuries and TGF-beta 1 production by HGF in attenuation of murine diabetic nephropathy. Am J Physiol Renal Physiol 286: F134-F143, 2004.

48. Schiffer M, Schiffer LE, Gupta A, Shaw AS, Roberts IS, Mundel P and Böttinger EP: Inhibitory smads and TGF- $\beta$ signaling in glomerular cells. J Am Soc Nephrol 13: 2657-2666, 2002.

49. Mu W, Ouyang X, Agarwal A, Zhang L, Long DA, Cruz PE, Roncal CA, Glushakova OY, Chiodo VA, Atkinson MA, et al: IL-10 suppresses chemokines, inflammation, and fibrosis in a model of chronic renal disease. J Am Soc Nephrol 16: 3651-3660, 2005.

50. Lewko B and Stepinski J: Hyperglycemia and mechanical stress: Targeting the renal podocyte. J Cell Physiol 221: 288-295, 2009.

51. Kato M, Putta S, Wang M, Yuan H, Lanting L, Nair I, Gunn A, Nakagawa Y, Shimano H, Todorov I, et al: TGF-beta activates Akt kinase through a microRNA-dependent amplifying circuit targeting PTEN. Nat Cell Biol 11: 881-889, 2009.

52. Kato M, Yuan H, Xu ZG, Lanting L, Li SL, Wang M, Hu MC, Reddy MA and Natarajan R: Role of the Akt/FoxO3a pathway in TGF-beta1-mediated mesangial cell dysfunction: a novel mechanism related to diabetic kidney disease. J Am Soc Nephrol 17: 3325-3335, 2006.

53. Huber TB, Hartleben B, Kim J, Schmidts M, Schermer B, Keil A, Egger L, Lecha RL, Borner C, Pavenstädt H, et al: Nephrin and $\mathrm{CD} 2 \mathrm{AP}$ associate with phosphoinositide 3-OH kinase and stimulate AKT-dependent signaling. Mol Cell Biol 23: 4917-4928, 2003.

54. Sanz AB, Sanchez-Niño MD, Ramos AM, Moreno JA, Santamaria B, Ruiz-Ortega M, Egido J and Ortiz A: NF-kappaB in renal inflammation. J Am Soc Nephrol 21: 1254-1262, 2010. 